\title{
Molecular Docking Analysis of Chloroquine and Hydroxychloroquine and Design of Anti-SARS-CoV2 Protease Inhibitor
}

\author{
Usman Abdulfatai $^{1}$, Adamu Uzairu ${ }^{1}$, Gideon Adamu Shallangwa ${ }^{1} \&$ Sani Uba $^{1}$ \\ ${ }^{1}$ Department of Chemistry, Ahmadu Bello University P.M.B. 1044, Zaria-Nigeria \\ Correspondence: Usman Abdulfatai, Department of Chemistry, Ahmadu Bello University P.M.B. 1044, \\ Zaria-Nigeria.
}

Received: August 21, 2020

Accepted: September 25, 2020

Online Published: September 28, 2020

doi:10.5539/mas.v14n10p52

URL: https://doi.org/10.5539/mas.v14n10p52

\begin{abstract}
In this present investigation, simulated molecular docking study of chloroquine and hydroxychloroquine compounds were investigated on the SARS-CoV2 enzyme to determine the types of amino acids responsible for the biochemical reaction at the binding site. A structure-based docking design technique was explored in designing a novel derivative of chloroquine for the treatment and management of new COVID 19 disease. To achieve this, the molecular docking simulation method was used to investigate the level of chloroquine and hydroxychloroquine (Drugs presently under clinical trial) interactions on SARS-CoV2 enzyme (a causative agent of COVID 19 disease). Chloroquine and hydroxychloroquine which has been debated as drugs for the management of COVID 19 were subjected to molecular docking analysis, and the binding energies generated were found to be $-6.1 \mathrm{kcal} / \mathrm{mol}$ and $-6.8 \mathrm{kcal} / \mathrm{mol}$ respectively. Moreover, novel 2-((4-((7-chloroquinolin-4 $\mathrm{yl})$ amino)pentyl)((methylamino)methyl)amino) ethan-1-ol as an anti-SARS-CoV2 protease was designed through the structural modification of hydroxychloroquine. The binding energy of this drug candidate was found to be $-6.9 \mathrm{kcal} / \mathrm{mol}$. This novel drug was found to formed hydrogen and conventional interactions with the binding site of SARS-CoV2 protease through amino acids such as Glutamic acid (GLU166), Glycine (GLY143), Phenylalanine (PHE140), Asparagine (ASN142), Histidine (HIS163), His (HIS172, HIS41, HIS163), Leucine (LEU41, LEU27), Glycine (GLY143), Glutamine (GLN189), Methionine (MET49, MET165), Serine (SER 46), Cysteine (CYS145) and Threonine (THR25). With this binding energy, this new drug candidate could bind better to the human SARS-CoV2 protease' binding site. This research provides a clue for other scientists on various ways of designing and identify the types of amino acids that may be responsible for biochemical action on SARS-CoV2 protease.
\end{abstract}

Keywords: Molecular docking simulation, COVID 19, SARS-CoV2, CADD

\section{Introduction}

The Severe Acute Respiratory Syndrome - Coronavirus 2 (SARS-CoV2) enzyme has been reported as the possible agent of Coronavirus disease-19 (COVID-19), the novel respiratory disease. Toward the last month of 2019 in Wuhan (a city in China), this virus was first reported before it was rapidly identified in other cities in China and in extension to many countries of the world [1-3]. The severity of this virus has necessitated the world health organization to refer to it as "Global pandemic". As of $20^{\text {th }}$ August 2020, the WHO reported 22256220 confirmed cases, with 782456 (3.52\%) death [3-5]. Apart from china which has contained the virus, other world countries have been unable to fight the spread of the virus, with the USA has the highest number of confirmed cases. To contain the spread of this virus, some preventive measures have been proposed by the WHO; social distance, regular handwashing with soap, avoids face robbing and cough and sneeze responsibly [3-5].

As of the time of writing this article, there have no known treatments/cure for this novel disease other than supportive care. But in in-vitro studies, some drugs have shown to be reliable for the treatment of this novel virus. And in-vivo (pre-clinical) studies have since commenced for some of these drugs (Chloroquine and hydroxychloroquine) across the world [3,4]. The knowledge of binding energy, the active site of these drug candidates against SARS-CoV2 will be important in the field of pharmaceutical drug design. Since this will also enhance our knowledge of how drug inhibits the virus from replicating [2-5]. Most of the pharmaceutical drug companies often use computational aided drug design (CADD) as one of the few important techniques of drug design and development. This hypothetical method is simple and fast and it has proven to be instrumental in 
designing better drugs for other ailments (Tactrin for Alzheimer's disease, Relenza for influenza, and Invirase for HIV protease inhibitor) [6-8]. Therefore, the objective of this study was to used molecular docking technique as an investigation tool to design and to search for better binding energy and excellent orientation between chloroquine/hydroxychloroquine and SARS-CoV2 (enzyme causing COVID 19).

\section{Materials and Methods}

\subsection{Ligands and Receptors}

Using Chemdraw, two-dimensional structures of Chloroquine and hydroxychloroquine (Ligands) were sketched and subsequently converted to three-dimensional structures (Figure 1). The converted structures were geometrically optimized with the help of density functional theory algorithm of multifunctional Spartan 14 software [9]. Moreover, the causative receptor (SARS-CoV2) of COVID 19 enzyme (Figure 1) was carefully retrieved from http://www.rcsb.org/pdb and prepared by the addition of hydrogen, removal of water and other heteroatoms.

\subsection{Molecular Docking's Computational Procedure}

By exploring the flexible docking option of Autoduck vina docking software, SARS-CoV2 receptor and ligands were set into the X, Y and Z grid. For optimal docking efficiency, the search option was set at 100 per cent. The best binding energy was selected to search for the best ligand orientation in the SARS-CoV2 enzyme using Autodock program. For the docking investigation, last two conformers were utilized for every ligand-receptor complex, and the overall binding clusters were examined with a $2.0 \AA$ RMSD [10]. Using discovery studio visualizer, the ligand-receptor 3D complex binding interactions involving hydrophobic and hydrogen were viewed.
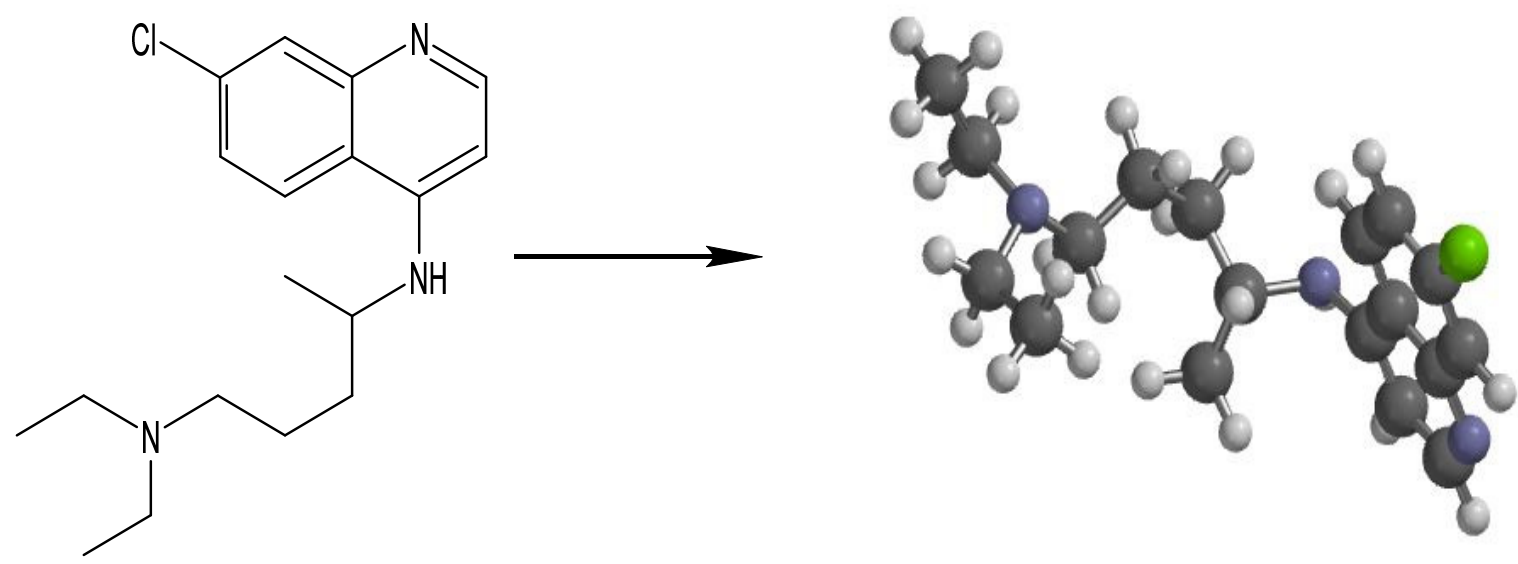

Chloroquine
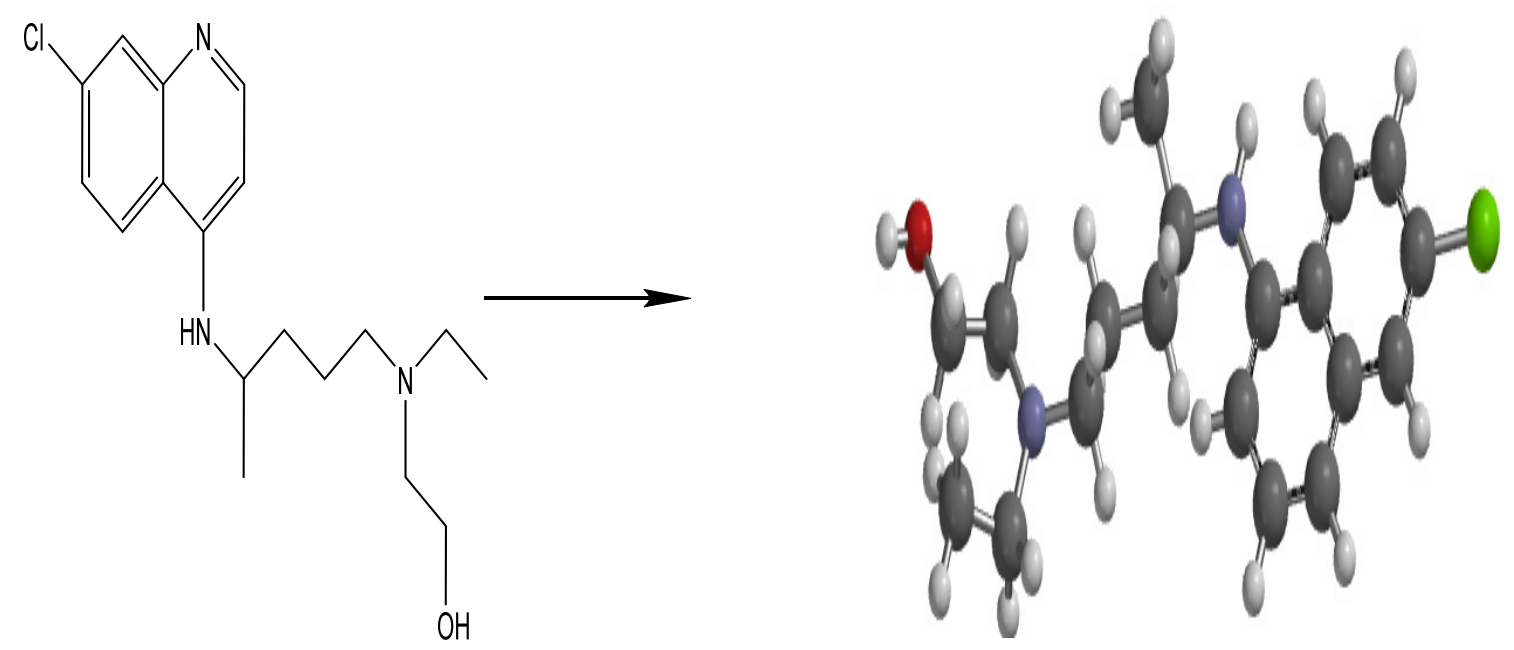

Hydroxychloroquine 


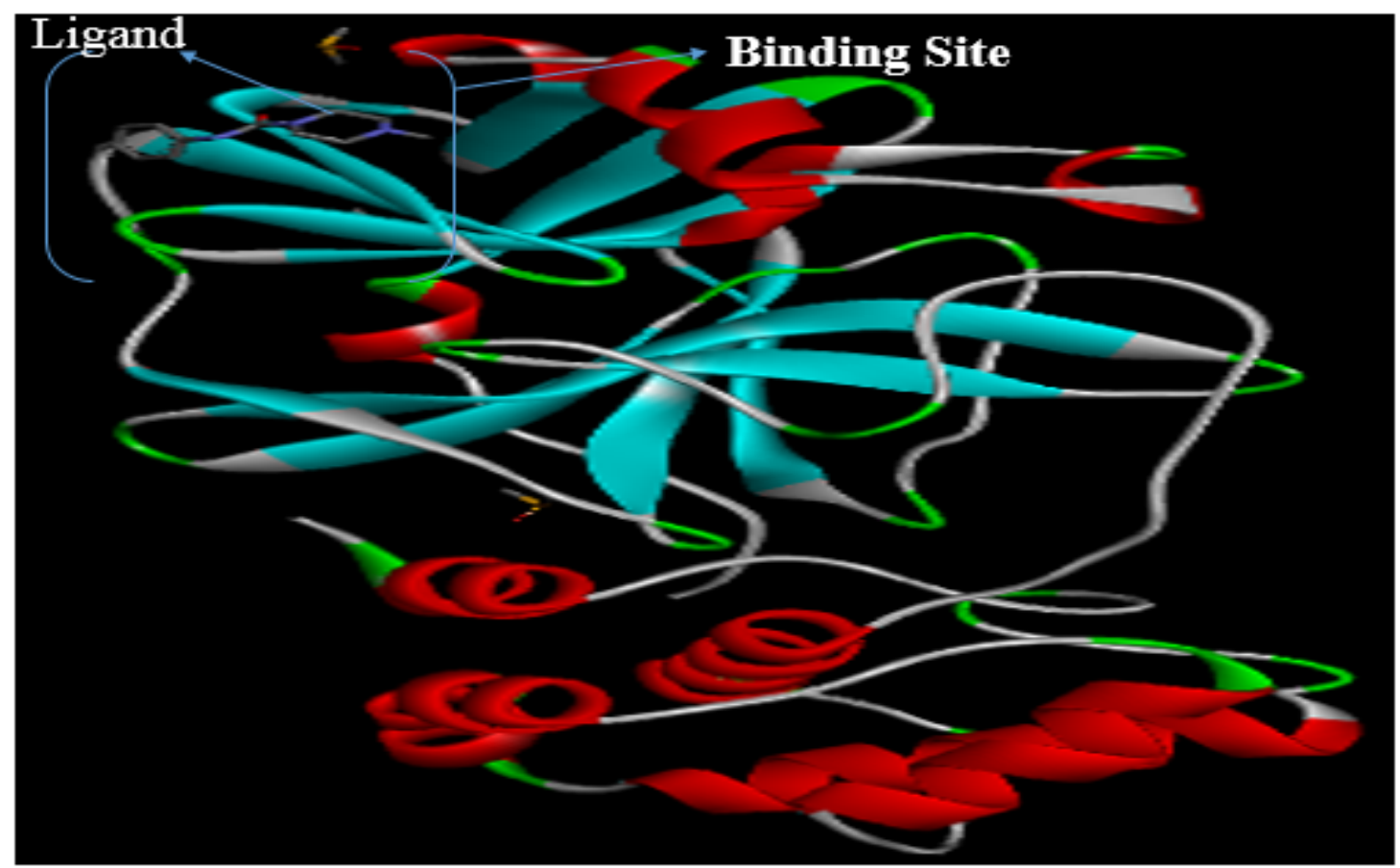

Figure 1. The 2D/3D structures of Chloroquine, Hydroxychloroquine and SARS-CoV2 enzyme

\section{Results and Discussion}

Simulated molecular docking study of chloroquine and hydroxychloroquine ligands were investigated on the SARS-CoV2 enzyme to determine the types of amino acids responsible for the biochemical reaction at the binding site. After the systematic simulation of these ligands on the protein receptor (Figure 1), it was confirmed that the two ligands were bounds to the receptor through its amino acids residues at the binding site. The chloroquine ligand formed binding energy of $-6.1 \mathrm{kcal} / \mathrm{mol}$ (and $3.67 \AA$ hydrogen bond length) by interacting with Valine (VAL73, VAL36, VAL91, VAL18 and VAL68), Leucine (LEU89), Threonine (THR93), Histidine (HIS80), Alanine (ALA70), Aspartic acid (ASP92), Isoleucine (ILE78), Phenylalanine (PHE66), Asparagine (ASN63), Lysine (LYS90), Glycine (GLY79) and Tryptophan (TRP31) amino acid binding residues (Table 1), at the binding site of the SARS-CoV2 receptor. One of the derivatives of chloroquine, hydroxychloroquine was also revealed to the formed binding energy of $-6.8 \mathrm{kcal} / \mathrm{mol}$ (and 2.14, 2.16, 3.04 and $3.47 \AA$ hydrogen bond lengths) by forming interactions with some amino acids such as Glycine (GLY79), Isoleucine (ILE78), Tryptophan (TRP31), Valine (VAL91, VAL68 and VAL73), Aspartic acid (ASP92), Leucine (LEU89 and LEU67), His (HIS80), Lysine (LYS90), Phenylalanine (PHE66), Threonine (THR93) and Asparagine (ASN63) at the binding site of the SARS-CoV2 receptor (Table 1 and figure $2 \mathrm{~A}$ and $\mathrm{B}$ ).

Table 1. Structures of the newly designed anti- SARS-CoV2 compounds

\begin{tabular}{|c|c|c|c|c|c|}
\hline \multirow[b]{2}{*}{$\mathrm{S} / \mathrm{N}$} & \multirow[b]{2}{*}{ Ligand } & \multirow[b]{2}{*}{ Drug Target } & \multirow[b]{2}{*}{$\begin{array}{l}\text { Binding Energy } \\
(\mathrm{Kcal} / \mathrm{mol})\end{array}$} & \multicolumn{2}{|l|}{ Hydrogen bonding } \\
\hline & & & & $\begin{array}{l}\text { Binding Amino Acid } \\
\text { Residues }\end{array}$ & Bond Length $(\AA)$ \\
\hline 1 & Chloroquine & SARS-CoV2 & -6.1 & VAL A: 73 & 3.67 \\
\hline 2 & hydroxychloroquine & SARS-CoV2 & -6.8 & $\begin{array}{l}\text { GLY A: } 79, \text { ILE A: } 78, \\
\text { TRP A: } 31 \text { and ASN A: } 63\end{array}$ & $\begin{array}{l}2.14,2.16,3.04 \\
\text { And } 3.47\end{array}$ \\
\hline 3 & $\begin{array}{l}\text { Designed novel } \\
\text { compound }\end{array}$ & SARS-CoV2 & -6.9 & $\begin{array}{l}\text { GLU A: } 166, \text { GLY A: } 143 \text {, } \\
\text { PHE A: } 140, \text { ASN A: } 142 \\
\text { and HIS A: } 163\end{array}$ & $\begin{array}{l}3.09,2.33,2.55 \\
3.60 \text { and } 3.78(\AA)\end{array}$ \\
\hline
\end{tabular}




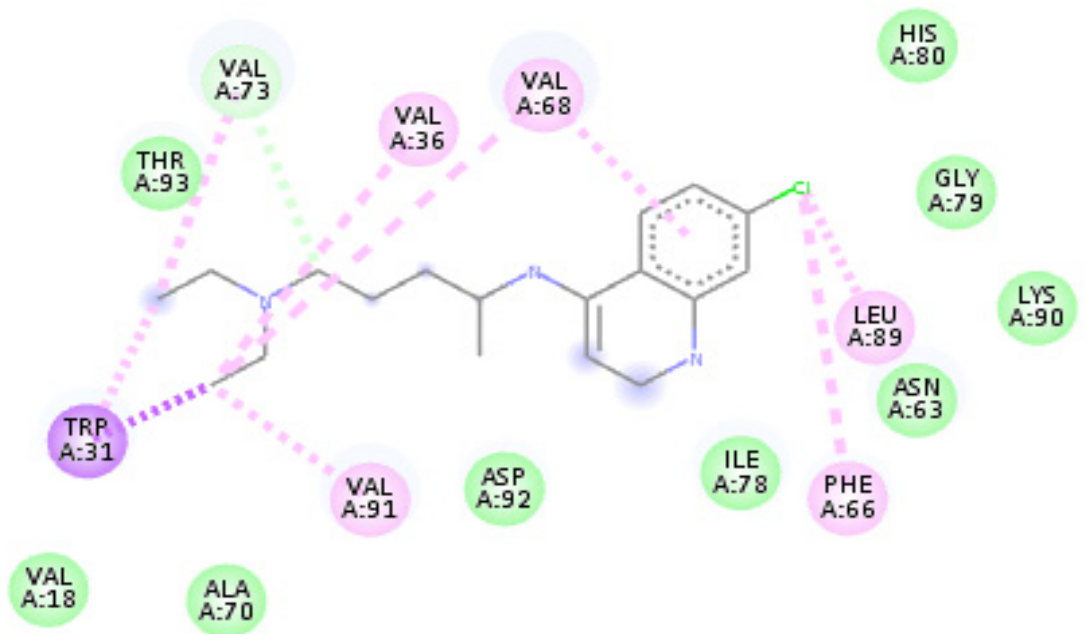

\section{Interactions}
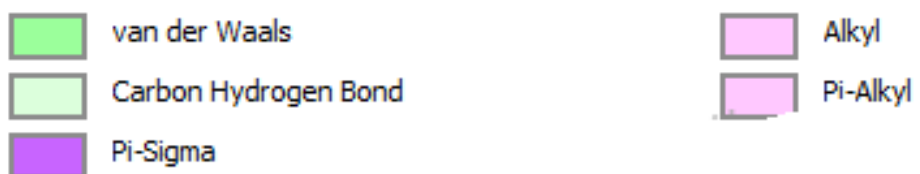

Figure 2A. 2D structure of chloroquine- SARS-Cov-2 receptor complex 


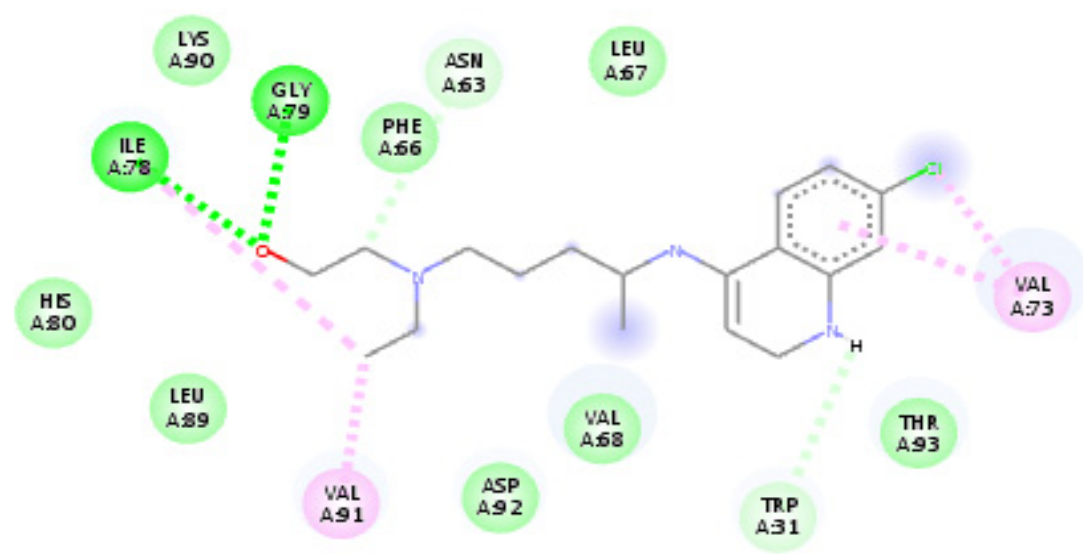

\section{Interactions}

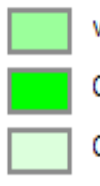

van der Waals

Conventional Hydrogen Bond

Pi-Donor Hydrogen Bond

Carbon Hydrogen Bond

Alkyl

Pi-Alkyl

Figure 2B. 2D structure of hydroxychloroquine- SARS-Cov-2 receptor complex

\subsection{Designed Anti-SARS-CoV2 Drug Candidate Analysis}

In this present investigation, a structure-based docking design technique was explored in designing a novel derivative of chloroquine for the treatment and management of this novel COVID-19 disease. To achieve this, hydroxychloroquine was chosen as the design template due to its excellent binding energy (Table 1) and level of interactions (Figure 2B) than its co-ligand (chloroquine). After making some structural modifications to the chosen template, a drug candidate was designed (Figure 3). The 2-((4-((7-chloroquinolin-4 yl)amino)pentyl)((methylamino)methyl)amino) ethan-1-ol drug candidate was designed and was found to have a
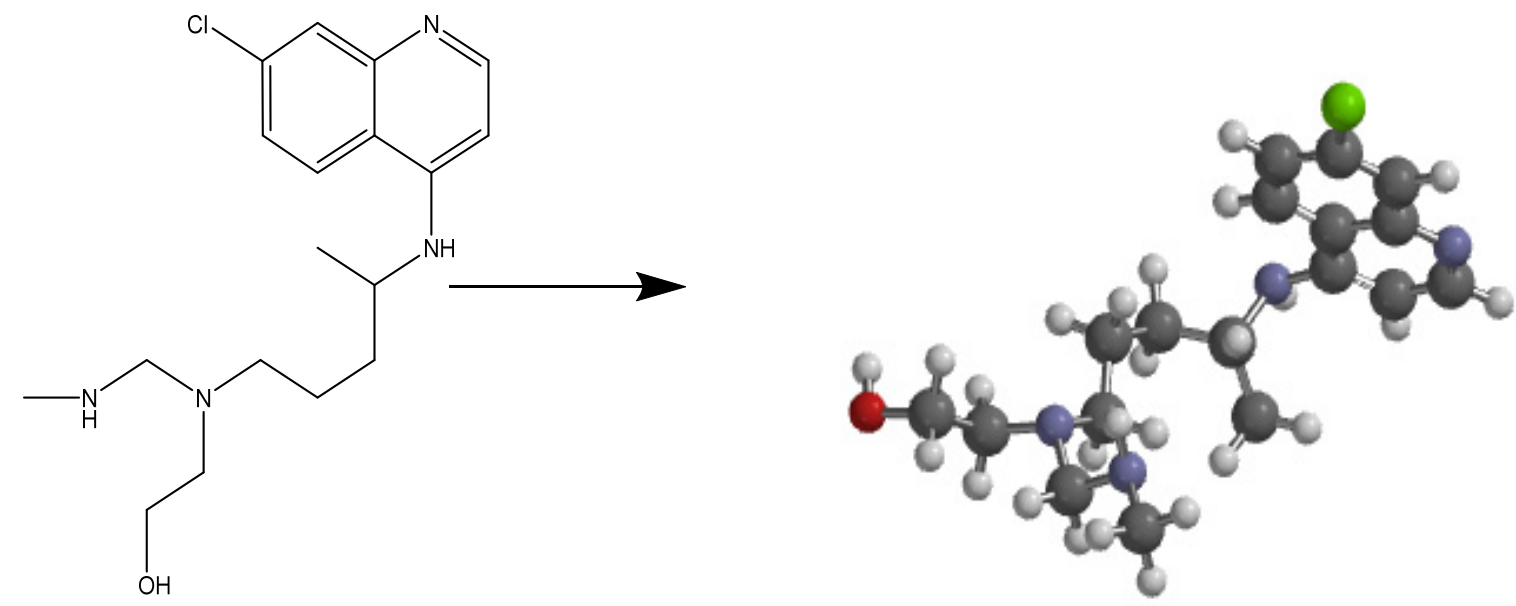

Figure 3. 2D structure of the designed drug candidate, 2-((4-((7-chloroquinolin-4 yl)amino)pentyl)((methylamino)methyl) amino)ethan-1-ol 
better binding energy of $-6.9 \mathrm{kcal} / \mathrm{mol}$ (Table 1) than chloroquine $(-6.1 \mathrm{kcal} / \mathrm{mol})$ and hydroxychloroquine $(-6.8$ $\mathrm{kcal} / \mathrm{mol}$ ). This novel hypothetical inhibitor formed hydrogen bonds and hydrogen bond lengths of 3.09, 2.33, 2.55, 3.60 and 3.78 $\AA$ by reacting with amino acid residues such as Glutamic acid (GLU166), Glycine (GLY143), Phenylalanine (PHE140), Asparagine (ASN142), Histidine (HIS163), His (HIS172, HIS41, HIS163), Leucine (LEU41, LEU27), Glycine (GLY143), Glutamine (GLN189), Methionine (MET49, MET165), Serine (SER 46), Cysteine (CYS145), Threonine (THR25) of the enzyme's binding site (Figure 4) respectively. This new structurally designed inhibitor also forms other conventional interactions with amino acids such as Histidine (HIS41), Methionine (MET49 and MET165) and Cysteine (CYS145). Furthermore, a closed observation of the interaction indicated that substituents like $\mathrm{H}(\mathrm{H}-\mathrm{O}), \mathrm{O} \quad(\mathrm{C}-\mathrm{O}), \quad \mathrm{C} \quad(-\mathrm{CHO})$ and $\mathrm{C} \quad(-\mathrm{CH} 2)$ of 2-((4-((7-chloroquinolin-4 yl)amino)pentyl)((methylamino)methyl)amino) ethan-1-ol formed some conventional and carbon-hydrogen bonds with SARS-Cov-2 Protease through GLU166, GLY143, PHE140, ASN142 and HIS163 amino acid (Figure 4).

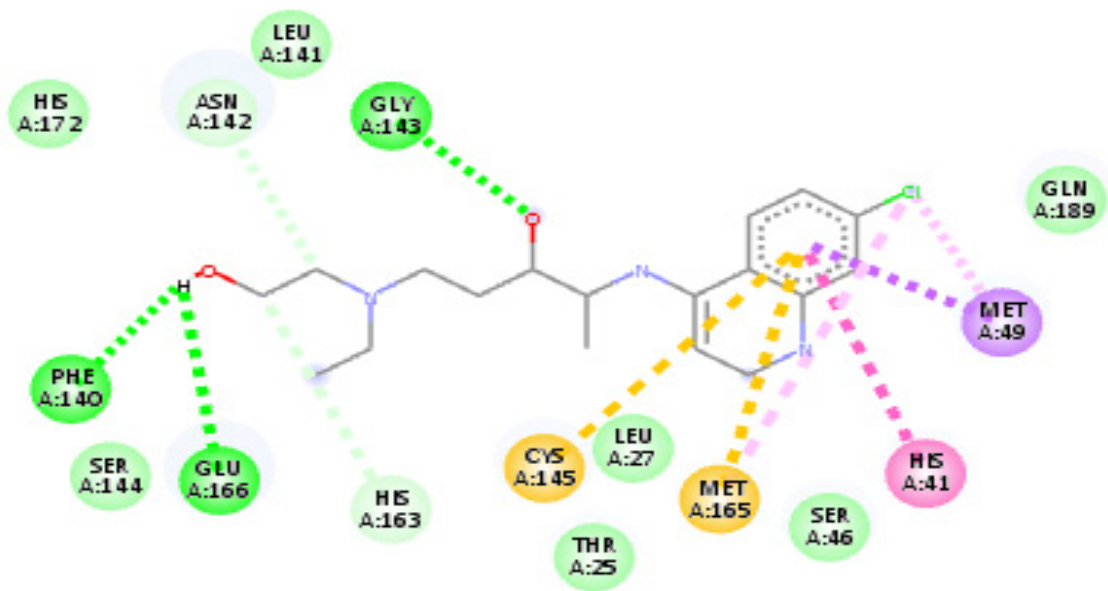

\section{Interactions}
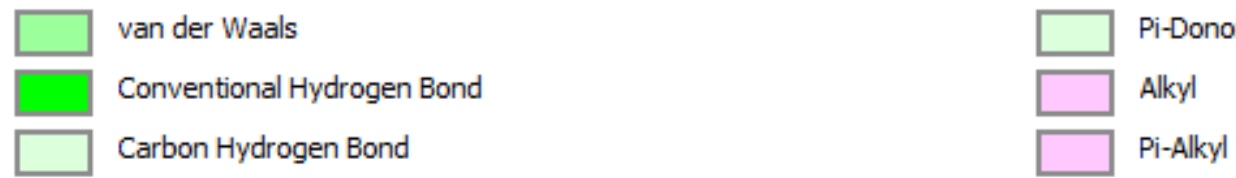

Figure 4. 2D structure of the designed drug candidate- SARS Cov2 receptor complex

\section{Conclusion}

Simulated molecular docking study of chloroquine and hydroxychloroquine ligands were investigated on the SARS-CoV2 enzyme to determine the types of amino acids responsible for the biochemical reaction at the binding site. Chloroquine and hydroxychloroquine which has been debated as drugs for the treatment of COVID 19 were subjected to molecular docking analysis, and the binding energies generated were found to be -6.1 $\mathrm{kcal} / \mathrm{mol}$ and $-6.8 \mathrm{kcal} / \mathrm{mol}$ respectively. These two high binding energies revealed the binding strength of these two compounds against the SARS-CoV2 protease. Moreover, novel 2-((4-((7-chloroquinolin-4 yl) amino)pentyl)((methylamino)methyl)amino) ethan-1-ol as an anti-SARS-CoV2 protease was designed through the structural modification of hydroxychloroquine. The binding energy of this drug candidate was found to be 
$-6.9 \mathrm{kcal} / \mathrm{mol}$. This novel drug was found to bind and form hydrogen and conventional bonding with the binding site of SARS-CoV2 protease through amino acids such as Glutamic acid (GLU166), Glycine (GLY143), Phenylalanine (PHE140), Asparagine (ASN142), Histidine (HIS163), His (HIS172, HIS41, HIS163), Leucine (LEU41, LEU27), Glycine (GLY143), Glutamine (GLN189), Methionine (MET49, MET165), Serine (SER 46), Cysteine (CYS145) and Threonine (THR25). With this binding energy, this new drug candidate could bind better to the human SARS-CoV2 protease' binding site. This research provides a clue for other scientists on various ways of identifying the types of amino acids that may responsible for biochemical protein action on SARS-CoV2 protease.

\section{Competing interests}

None

Funding

None

\section{References}

Chatre, C., Roubille, F., Vernhet, H., Jorgensen, C., \& Pers, Y. M. (2018). Cardiac complications attributed to chloroquine and hydroxychloroquine: a systematic review of the literature. Drug safety, 41(10), 919-931. https://doi.org/10.1007/s40264-018-0689-4

Guo, Y. R., Cao, Q. D., Hong, Z. S., Tan, Y. Y., Chen, S. D., Jin, H. J., \& Yan, Y. (2020). The origin, transmission and clinical therapies on coronavirus disease 2019 (COVID-19) outbreak-an update on the status. Military Medical Research, 7(1), 1-10. https://doi.org/10.1186/s40779-020-00240-0

Hehre, W. J., \& Huang, W. W. (1995). Chemistry with computation: An introduction to SPARTAN. Wavefunction, Inc.

Ahmad S, Hafeez A, Siddqui SA, Ahmad M, Mishra S. A Review of COVID-19 (Coronavirus Disease-2019) Diagnosis, Treatments and Prevention. EJMO 2020; 4(2):116-125.

Jorgensen, W. L. (2004). The many roles of computation in drug discovery. Science, 303(5665), 1813-1818. https://doi.org/10.1126/science.1096361

Kitchen, D. B., Decornez, H., Furr, J. R., \& Bajorath, J. (2004). Docking and scoring in virtual screening for drug discovery: methods and applications. Nature reviews Drug discovery, 3(11), 935-949. https://doi.org/10.1038/nrd1549

Langer, T., \& Hoffmann, R. D. (2001). Virtual screening an effective tool for lead structure discovery. Current pharmaceutical design, 7(7), 509-527. https://doi.org/10.2174/1381612013397861

Lu, R., Zhao, X., Li, J., Niu, P., Yang, B., Wu, H., \& Bi, Y. (2020). Genomic characterisation and epidemiology of 2019 novel coronavirus: implications for virus origins and receptor binding. The Lancet, 395(10224), 565-574. https://doi.org/10.1016/S0140-6736(20)30251-8

Chen, J., Ping, L., \& Li, L. (2020). Preliminary study of hydroxychloroquine sulfate in treating common coronavirus disease (COVID-19) patients in 2019. Journal of Zhejiang University (Medical Science).

Sambasivarao, S. V., Roberts, J., Bharadwaj, V. S., Slingsby, J. G., Rohleder, C., Mallory, C., \& Maupin, C. M. (2014). Acetylcholine Promotes Binding of $\alpha$-Conotoxin MII at $\alpha 3 \beta 2$ Nicotinic Acetylcholine Receptors. Chembiochem, 15(3), 413-424. https://doi.org/10.1002/cbic.201300577

\section{Copyrights}

Copyright for this article is retained by the author(s), with first publication rights granted to the journal.

This is an open-access article distributed under the terms and conditions of the Creative Commons Attribution license (http://creativecommons.org/licenses/by/4.0/). 